\title{
Movilidad residencial y política de redensificación: el área central de la Ciudad de México*
}

\author{
CATHERINE PAQUETTE VASSALLI** Y DANIEL DELAUNAY*** \\ ** Investigadora del Instituto Francés de Investigación para el Desarrollo (IRD). \\ *** Director de Investigación, investigador del Instituto Francés de Investigación para el Desarrollo (IRD).
}

\begin{abstract}
Latin American metropoli reveal an increasing interest in urban policies of redensification of central area which have suffered population loss over several decades. However, prior implementing measures to retain existing residents and attract new ones, it is necessary not to be restricted to mere evidence of a reduction of the number of residents: a more profound diagnosis of the population loss process is required. Within this diagnosis, it is necessary to analyze migratory patterns to and from the centre, with the aim of revealing, among other variables, the profiles of the inhabitants who leave the centre, and where they move. It is only from these variables that it is possible to design adequate urban policies. Also, as the Mexico City case illustrates, it is very important to develop global urban strategies for the whole of the metropolis.
\end{abstract}

KEYWORDS: Mexico City, central areas, redensification, population loss, residential mobility.

RESUMEN Las metrópolis de América Latina muestran un interés creciente para la implementación de políticas urbanas de redensificación en sus áreas centrales, espacios que sufren pérdidas de población muy importantes desde hace varias décadas. Sin embargo, antes de implementar medidas para retener a los residentes actuales y atraer a nuevos, resulta indispensable no limitarse a la mera evidencia de una disminución del número de residentes: se tiene que establecer un diagnóstico a profundidad referente al proceso de despoblamiento. Dentro de este diagnóstico, resulta necesario analizar los movimientos migratorios hacia y a partir del centro, con el objetivo de conocer, entre otras variables, cuáles son los perfiles de los habitantes que abandonan el centro y hacia dónde se dirigen. Es solo a partir de estas variables que se podrán diseñar políticas urbanas adecuadas. Además, tal y como lo muestra el caso aquí estudiado de la Ciudad de México, es sumamente importante desarrollar estrategias urbanas globales para toda la metrópoli.

PALABRAS CLAVE: Ciudad de México, áreas centrales, redensificación, despoblamiento, movilidad residencial

\footnotetext{
Artículo elaborado en el marco del proyecto de investigación "Política urbana y movilidades: el caso del área central de la Ciudad de México” (2005-2008; IRD/UMR 201 Développement et Sociétés y El Colegio de México/ Centro de Estudios Demográficos, Urbanos y Ambientales).

Recibido el 3 de octubre de 2008, aprobado el 12 de junio de 2009.

Correspondencia: Dra. Catherine Paquette Vassalli, Institut de Recherche pour le Développement, UR 29 Urbi Environnement Urbain, Centre de recherche d'Ile-de-France 32, avenue Henri Varagnat, F - 93143 Bondy cedex. E-mail : catherine.paquette@ird.fr. Dr. Daniel Delaunay, Institut de Recherche pour le Développement, UMR 201 Développement et Sociétés, Université de Paris 1-IRD, Campus du Jardin tropical de Paris, 45 bis, avenue de la Belle Gabrielle, 94736 Nogent-sur-Marne, France. E-mail: daniel.delaunay@ird.fr.
} 


\section{Introducción}

Las áreas centrales de las metrópolis de América Latina comparten numerosas evoluciones comunes: una de ellas, iniciada hace varias décadas, es la tendencia al despoblamiento. Aunque esta dinámica haya perdido intensidad, sigue siendo hoy en día actual. Como en el pasado, se debe a una desconcentración generalizada de población que se produce desde la zona de urbanización más antigua y mejor consolidada, en beneficio de contornos urbanos más recientes y de las periferias, generalmente de rápida expansión. Hasta hoy, dos metrópolis han intentado establecer estrategias de gran amplitud para frenar este proceso preocupante. Santiago de Chile es la primera de ellas, con un programa de repoblamiento lanzado desde principios de los años 90, basado en gran parte en la producción, en el centro, de una oferta de viviendas en acceso a la propiedad muy subvencionadas (Paquette, 2005). La Ciudad de México, específicamente el Distrito Federal (DF), constituye la segunda experiencia de este tipo en América Latina. La política denominada "Bando 2", implementada entre 2001 y 2006, consistió en restringir la construcción de nuevos conjuntos habitacionales casi exclusivamente dentro de la catalogada "Ciudad Central"; sector céntrico muy amplio y heterogéneo, conformado por cuatro de la diez y seis delegaciones político-administrativas que constituyen el Distrito Federal (ver figura 1). Con esta acción, se pretendía interrumpir el despoblamiento de esta área y favorecer la llegada de nuevos habitantes, limitando al mismo tiempo el crecimiento urbano formal en las periferias del Distrito Federal que contaban todavía con suelo no urbanizado. Esta política tenía una connotación social marcada, ya que pretendía otorgar a los hogares de bajos ingresos la posibilidad de vivir en este espacio (Programa General de Desarrollo Urbano, 2003). Cabe señalar que hasta la fecha, los esfuerzos para intervenir en el área central de la ciudad se habían concentrado estrictamente en el Centro Histórico, un espacio mucho más restringido que la Ciudad Central y con características patrimoniales, en lo cual se implementaron, desde inicios de los años noventa en particular, numerosas medidas a favor de su revitalización, incluyendo también, para el periodo reciente, intentos por atraer nuevos residentes (Paquette, 2006). 


\section{LA ZONA METROPOLITANA DE LA CIUDAD DE MÉXICO Y LA POLÍTICA DEL "BANDO 2"}

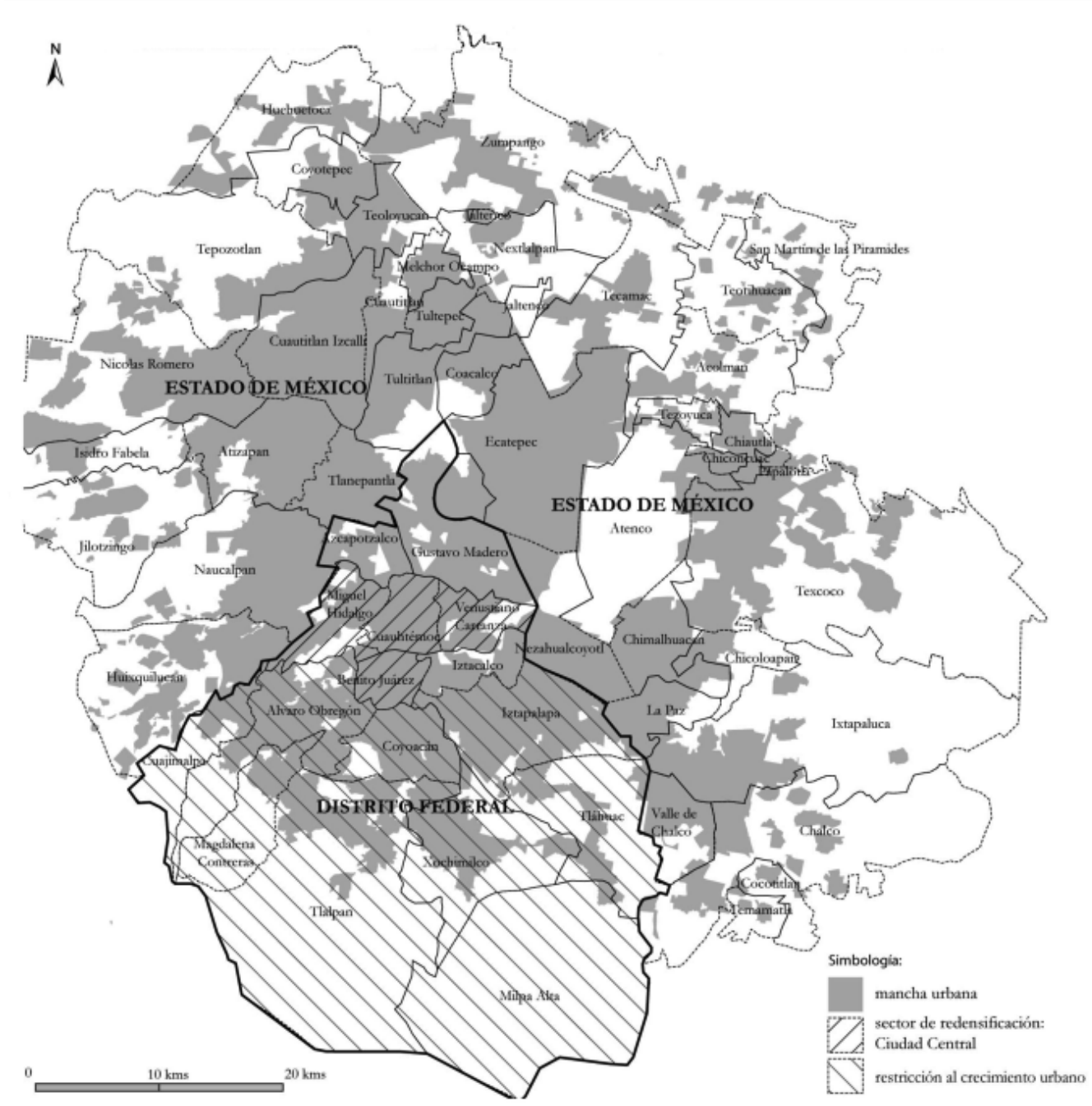

Fuente: Elaboración propia en base al Programa General de Desarrollo Urbano del DF 2001 - 2006

En Santiago de Chile y en la Ciudad de México, el éxito de las estrategias de redensificación de lo que podríamos calificar como el centro ampliado de la ciudad ha rebasado todas las expectativas: se produjo un regreso masivo de la construcción de nuevos conjuntos habitacionales en estos espacios, mismo que está siendo cuestionado: por una parte, a pesar de que se dio un verdadero boom inmobiliario, las metas en materia de repoblamiento no se han alcanzado (Paquette, 2005; 2008); por otra parte, aparecieron cambios, sutiles por cierto pero reales, en el tipo de poblamiento de las áreas objeto de estas políticas, en particular, con la llegada de residentes de nivel socio-económico más elevado (Contreras, 2005; Marcadet, 2007). En la Ciudad de México, cabe destacar sin embargo la producción importante de viviendas populares en las cuatro delegaciones centrales del Distrito Federal, a través de la acción del Instituto de la Vivienda del DF (Tamayo, 2007). 
En esta metrópoli, la política de redensificación se decretó de manera repentina, sin contar con un diagnóstico previo acerca del proceso de despoblamiento que fuera más allá de la evidencia de las pérdidas de población ocurridas desde hacía varias décadas y medidas por cada censo (la Ciudad Central perdió 1,2 millones de residentes entre 1970 y el 2000); este balance, no obstante, era primordial de hacer para determinar las medidas que se tenían que establecer con la finalidad de lograr los objetivos planteados: detener las salidas; atraer nuevos residentes y conservar las oportunidades para los hogares de bajos ingresos. Un diagnóstico de este tipo es sumamente complejo de realizar, dado que las causas del despoblamiento tienen que ver con numerosos factores, como son, entre otros: los cambios de uso de suelo; el costo y la disponibilidad de suelo; el nivel de las rentas; las características del parque habitacional disponible; la demanda habitacional existente; así como ciertos cambios demográficos y también societales. El objetivo de este artículo es centrarnos en una de estas dimensiones que consideramos indispensable de analizar en el contexto de una política de redensificación en lo residencial: la movilidad residencial entre la ciudad central y el resto de la zona metropolitana. Estas migraciones intrametropolitanas pueden ser analizadas a detalle en el periodo inmediatamente anterior a la aplicación del "Bando 2" (1995-2000) con los resultados del Censo de Población 2000, en el cual se introdujo por primera vez una pregunta referente al municipio de residencia de los individuos cinco años antes. Estos nuevos datos nos permiten, entre otros, acercarnos al perfil y a los destinos de los individuos que dejaron de residir en el área central durante el periodo considerado, así como a las características principales de los que llegaron a este espacio. Este acercamiento a la movilidad residencial intrametropolitana es clave de examinar y constituye sin lugar a dudas un primer paso ineludible (aunque no es el único, desde lejos) antes de diseñar cualquier tipo de política de repoblamiento. En momentos en que un número cada vez mayor de grandes ciudades latinoamericanas piensan en implementar estrategias de desarrollo urbano similares para sus áreas centrales, la importancia de estos diagnósticos migratorios previos tiene que ser subrayada.

\section{Un análisis de la movilidad residencial intrametropolitana en base a microdatos censa- les del 2000}

Este artículo presenta los resultados de un trabajo realizado a partir de la muestra al $10 \%$ del Censo de Población 2000, en particular, de la pregunta relativa al municipio de residencia de los individuos en 1995. Para llevar a cabo el análisis de los intercambios migratorios entre la Ciudad Central y el resto del espacio urbano metropolitano, hemos dividido la Zona Metropolitana de la Ciudad de México (ZMCM) en tres sectores concéntricos. El primero de ellos es la Ciudad Central, que corresponde a la porción de la Ciudad de México que ha sido objeto de una política de redensificación entre el 2001 y el 2006 y está constituida por las cuatro delegaciones centrales del DF. El "Distrito Federal” es el segundo sector de referencia: por ahí, entenderemos el resto del DF, correspondiendo a 12 delegaciones periféricas a la ciudad central. La Ciudad de México, que estaba anteriormente comprendida totalmente en el DF, empezó a rebasar sus límites desde los años 1940, extendiéndose al Estado de México. Para el año 2000, la zona metropolitana se extendía sobre una superficie de $1.500 \mathrm{~km}^{2}$, de los cuales, más de la mitad se situaba fuera del Distrito Federal, en los municipios conurbados del vecino Estado de México: mientras el DF contaba con 8,6 millones de habitantes, el resto de la aglomeración sumaba una población de 9,4 millones (Garza, 2000). El conjunto formado 
por los municipios conurbados, fuera del DF, es el tercer sector para nuestro análisis. Lo denominaremos "Estado de México" por comodidad, aunque este último esté lejos de reducirse a esta zona urbana de la Ciudad de México.

Aunque la división política DF-Estado de México, presente en la mayoría de los trabajos sobre la Zona Metropolitana de la Ciudad de México, es a menudo criticada (no existe ninguna ruptura espacial); el hecho de privilegiar esta separación en el marco de nuestro estudio tiene su justificación. Si bien es cierto que el límite entre las dos entidades no es una realidad en el plano físico, este cobra sentido en otros ámbitos, en materia de política y de gestión urbana sobre todo. De un lado y otro de la frontera política DF-Estado de México, las oportunidades para los hogares de bajo ingreso de encontrar dónde alojarse, y en particular, de volverse propietarios de una vivienda, no son en nada comparables. Las autoridades a cargo del DF han intentado desde hace más de una década limitar la urbanización en las periferias del sur del DF con un éxito relativo, pero ocurre todo lo contrario en la parte de la ciudad ubicada en el Estado de México. Desde hace mucho tiempo, las posibilidades de acceso a la vivienda son importantes y diversificadas: por una parte, en las urbanizaciones irregulares que siguen proliferando y en las cuales las familias pueden, mediante el pago de unos miles de pesos, hacerse propietarios de un lote del cual saben por experiencia que será un día regularizado (Huamán, 2005); por otra parte, en los gigantescos conjuntos de viviendas de interés social que fueron y siguen siendo construidos por promotores inmobiliarios en sectores periféricos cada vez más lejanos, en el marco de un esquema de política habitacional de tipo facilitadora, implementada en México a nivel federal a partir de los años noventa (Puebla, 2002). Estas urbanizaciones pueden sobrepasar las 10.000 unidades y están destinadas, mediante créditos otorgados por los organismos públicos de vivienda, a jefes de hogar asalariados que disponen de recursos en general superiores a cinco salarios mínimos (Boils, 2004). La "urbanización explosiva” de la periferia oriente de la aglomeración de México desde los años 1990 es ampliamente imputable a estos dos mercados habitacionales (Vega, 2003).

Debido a estas condiciones, la división DF-Estado de México, criticable en algunos puntos, existe pues en la realidad, por lo menos en cuestiones como la de movilidades residenciales. El análisis de las migraciones intraurbanas mostrará de hecho que no está desprovista de sentido, sino todo lo contrario.

\section{La ciudad central atrae población, pero los inmigrantes no compensan a los emigrantes}

Durante el periodo intercensal 1995-2000 (inmediatamente anterior a la implementación de la política de redensificación), las delegaciones de la ciudad central fueron relativamente atractivas, lo que no es sorprendente dado su localización. Benito Juárez fue por ejemplo la segunda entidad más atractiva, en valores relativos, de todo el Distrito Federal, con una proporción de inmigrantes intrametropolitanos del 11\% (ver figura 2 y cuadro 1). Una parte reducida (16\%) de los nuevos habitantes en esas cuatro delegaciones realizó un cambio de residencia dentro de este mismo espacio. Los cambios de residencia cuyos destinos fueron la ciudad central se debieron sobre todo a individuos procedentes del resto de la ciudad. 
Figura 2

INMIGRANTES INTRAMETROPOLITANOS:

POBLACIÓN RESIDENTE EN 2000 (DE MÁS DE CINCO AÑOS)

QUE VIVÍA EN OTRA UNIDAD POLÍTICO ADMINISTRATIVA DE LA ZMCM EN 1995

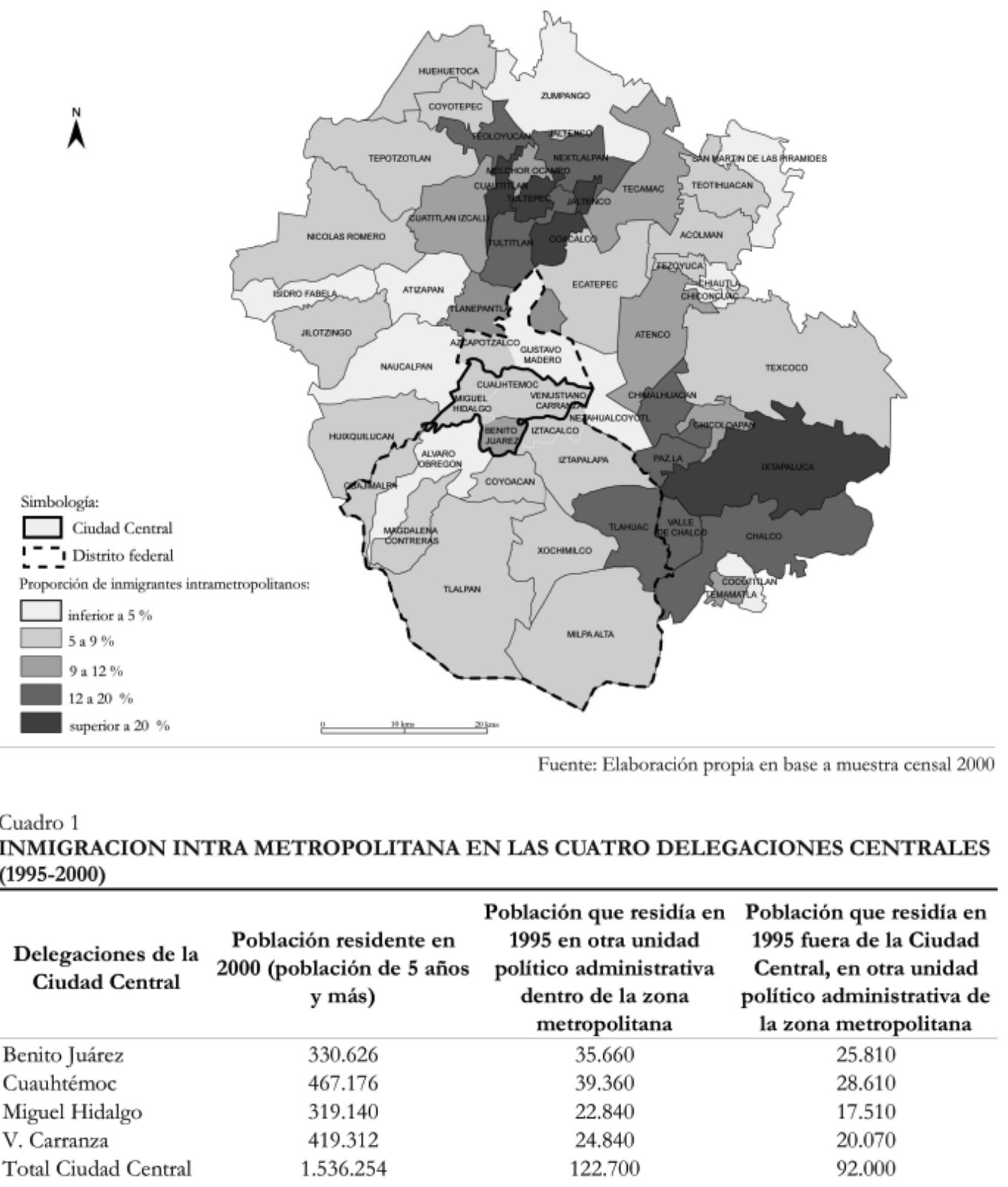

Fuente: Elaboración propia a partir del Censo de población 2000, muestra el $10 \%$

Estas llegadas no bastaron, sin embargo, para compensar las numerosas salidas. Entre 1995 y 2000, siguiendo una tendencia iniciada hace varias décadas, las delegaciones pertenecientes a la ciudad central han conocido una importante emigración, ampliamente superior, en valores relativos y también absolutos, a la de otras unidades político administrativas del resto de la 
zona metropolitana. Durante el intervalo de tiempo considerado, unos 200.000 individuos han dejado de residir en este espacio para irse a vivir en otra parte de la aglomeración. Se trata de un importante flujo, que representa el 14\% de la totalidad de las migraciones intra metropolitanas registradas en este periodo. Estos individuos que se fueron a otra unidad político administrativa de la zona metropolitana equivalen a un poco más del 11\% de la población de la ciudad central en 1995, lo que demuestra la amplitud del fenómeno (ver figura 3 y cuadro 2).

\section{Figura 3}

\section{EMIGRANTES INTRAMETROPOLITANOS:}

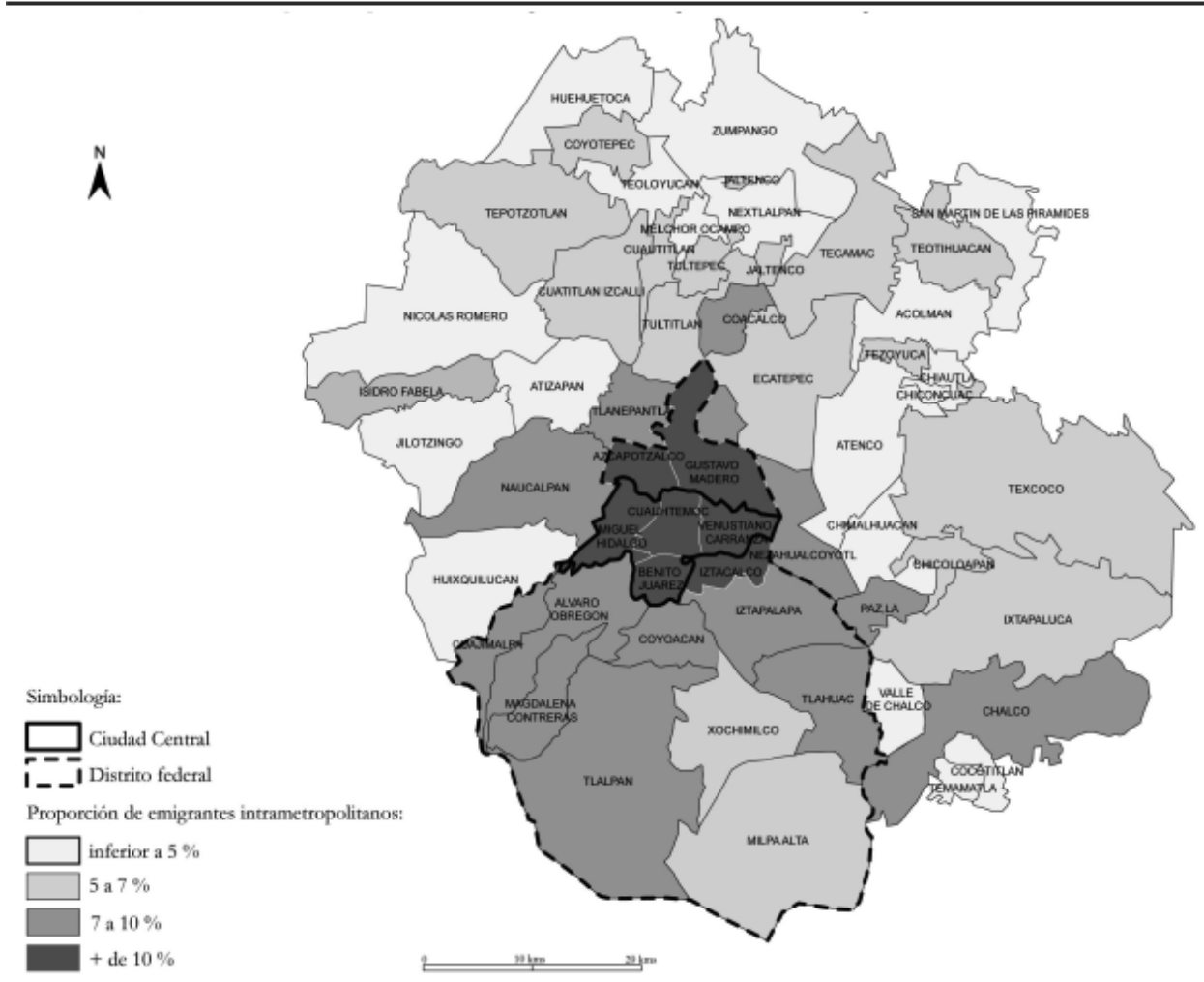

Fuente: Elaboración propia en base a muestra censal 2000 
Cuadro 2

EMIGRACION INTRA METROPOLITANA A PARTIR

DE LAS CUATRO DELEGACIONES CENTRALES (1995-2000)

\begin{tabular}{lccc}
\hline $\begin{array}{c}\text { Delegaciones de la } \\
\text { Ciudad Central }\end{array}$ & $\begin{array}{c}\text { Población } \\
\text { residente 1995 }\end{array}$ & $\begin{array}{c}\text { Población de cinco años } \\
\text { y más que reside en 2000 } \\
\text { en otra unidad político } \\
\text { administrativa dentro de } \\
\text { la zona metropolitana }\end{array}$ & $\begin{array}{c}\text { Población de cinco años } \\
\text { y más que reside en 2000 } \\
\text { en otra unidad político } \\
\text { administrativa dentro de } \\
\text { la zona metropolitana, } \\
\text { fuera de la Cd. Central }\end{array}$ \\
\hline Benito Juárez & 369.956 & 46.130 & 39.480 \\
Cuauhtémoc & 540.382 & 70.570 & 58.430 \\
Miguel Hidalgo & 364.398 & 45.190 & 39.850 \\
V. Carranza & 485.623 & 67.730 & 61.160 \\
Total Ciudad Central & 1.760 .359 & 229.620 & 198.920 \\
\hline
\end{tabular}

Fuente: Elaboración propia a partir del Censo de población 2000, muestra el $10 \%$

En total, las entradas a las cuatros delegaciones centrales representaron aproximadamente la mitad de las salidas, y este espacio registró un importante saldo migratorio negativo con el resto de la aglomeración (-100.000 individuos en un periodo de cinco años). Visto más a detalle, el déficit de la ciudad central con respecto al Estado de México (-65.910 individuos) equivale a más del doble del que tiene con el DF (-34.320): mientras las salidas del espacio central se repartieron de manera similar entre ambas entidades; en cambio, casi 7 de 10 nuevos residentes que llegaron al centro (procedentes de la zona metropolitana) provinieron del DF (ver cuadro 3).

Cuadro 3

INTERCAMBIOS DE POBLACION ENTRE LA CIUDAD CENTRAL Y EL RESTO DE LA ZONA METROPOLITANA (DF Y ESTADO DE MÉXICO)

\begin{tabular}{|c|c|c|c|c|c|}
\hline \multicolumn{2}{|c|}{ Salidas de la ciudad central } & \multicolumn{2}{|c|}{ Llegadas en la ciudad central } & \multicolumn{2}{|c|}{ Saldos migratorios } \\
\hline Cd. Central hacia DF & & DF hacia Cd. Central & & Cd. central-DF & \\
\hline & 102.210 & & 67.890 & & -34.320 \\
\hline Cd. central hacia el & & Edo. de México & & Cd. central-DF & \\
\hline Edo. de México & 96.710 & hacia Cd. central & 30.260 & & -66.450 \\
\hline $\begin{array}{l}\text { Total: Cd. Central } \\
\text { hacia zona }\end{array}$ & & $\begin{array}{l}\text { Total: zona } \\
\text { metropolitana hacia }\end{array}$ & & $\begin{array}{l}\text { Cd. central-zona } \\
\text { metropolitana }\end{array}$ & \\
\hline metropolitana & 198.920 & Cd. central & 98.150 & & -100.770 \\
\hline
\end{tabular}

Fuente: Elaboración propia a partir del Censo de población 2000, muestra el 10\%

Aunque la reflexión que hemos evocado trate de los intercambios migratorios dentro de la aglomeración, conviene subrayar que las aportaciones de población en la ciudad central desde el resto de la República Mexicana fueron del mismo modo limitadas: del total de individuos que llegaron a la zona metropolitana entre 1995 y 2000, solamente el $12 \%$ se instalaron en las cuatro delegaciones centrales, la mayoría (52,5\%), llegaron a los municipios conurbados del Estado de México. Esto confirma el importante cambio ocurrido en el patrón de migración a la Ciudad de México desde la provincia: hasta los ańos 1960, la mayor parte de los nuevos inmigrantes provenientes del resto del país llegaban directamente a la parte central del DF (Negrete, 2000), antes de reubicarse en otros sectores más periféricos, siguiendo el modelo 
identificado por Turner (1968). En la actualidad, y desde hace varias décadas, los nuevos habitantes que llegan de fuera de la zona metropolitana se asientan directamente en contornos urbanos periféricos y el centro no juega más un papel de receptor de la migración como antes (ver cuadro 4).

Cuadro 4

FLUJOS DE POBLACIÓN 1995-2000: INTRAMETROPOLITANOS Y DESDE EL EXTERIOR DE LA ZONA METROPOLITANA

Migración

Intra metropolitana: dentro de la Cd. Central

Cd. Central hacia Cd. Central

Número de

migrantes 30.700

Intra metropolitana: hacia la Cd. Central

DF hacia Cd. central

Estado de México hacia Cd. Central

Total

98.150

Intra metropolitana: desde la Cd. Central

Cd. Central hacia DF

Cd. Central hacia Estado de México

96.710

Total

198.920

Otras migraciones intra metropolitanas

DF hacia DF

DF hacia Estado de México

341.980

Estado de México hacia DF

105.680

Estado de México hacia Estado de México

393.050

Total

Migración procedente del exterior de la zona metropolitana

Provincia hacia $\mathrm{Cd}$ central

61.680

Exterior hacia Cd central

12.110

Provincia hacia DF

176.640

Provincia hacia Estado de México

263.300

Exterior hacia DF

13.790

Exterior hacia Estado de México

13.330

Total

202.640

Total migracion hacia la Cd. Central

1.407.330

Total migraciones intra metropolitanas

$\mathbf{5 4 0 . 8 5 0}$

Total migraciones desde fuera del área metropolitana

Fuente: Elaboración propia a partir del Censo de población 2000, muestra el 10\% 


\section{Las periferias populares del oriente de la zona metropolitana: destino importante de los individuos que salieron de la ciudad central}

Un poco más de la mitad de las migraciones intraurbanas a partir de la ciudad central tuvieron como destino entidades político-administrativas limítrofes a ésta y constituyeron movilidades residenciales que se operaron a una escala territorial finalmente bastante limitada.

Fuera de estos cambios de residencia, se puede observar que los movimientos migratorios teniendo como punto de partida la ciudad central se orientaron ampliamente hacia las periferias de urbanización popular de la parte oriente de la aglomeración: zonas de urbanización ya antigua y consolidada, por una parte, y sectores urbanizados más recientemente, muy marcados por la presencia de grandes conjuntos de viviendas de interés social, por otra parte (ver cuadro 5).

Cuadro 5

PRINCIPALES DESTINOS DE LOS MIGRANTES INTRA METROPOLITANOS A PARTIR DE LA CIUDAD CENTRAL Y SUS CARACTERÍSTICAS

\begin{tabular}{|c|c|c|c|c|c|c|}
\hline $\begin{array}{l}\text { Unidad político } \\
\text { administrativa }\end{array}$ & $\begin{array}{l}\text { Emigrantes } \\
\quad \text { intra } \\
\text { métropolitanos } \\
\text { 1995-2000 }\end{array}$ & $\begin{array}{l}\% \text { del total de } \\
\text { emigracion } \\
\text { intra } \\
\text { metropolitana } \\
1995-2000 \text { a } \\
\text { partir de la cd. } \\
\text { Central }\end{array}$ & $\begin{array}{l}\text { Tipo de unidad } \\
\text { político- } \\
\text { administrativa }\end{array}$ & $\begin{array}{c}\% \text { de la } \\
\text { población } \\
\text { ocupada con } \\
\text { menos de dos } \\
\text { salarios } \\
\text { mínimos* }\end{array}$ & $\begin{array}{c}\% \text { de la } \\
\text { población } \\
\text { ocupada con } \\
\text { más de cinco } \\
\text { salarios } \\
\text { mínimos** }\end{array}$ & $\begin{array}{c}\text { Viviendas de } \\
\text { intéres social } \\
\text { construidas entre } \\
1995 \text { y } 2000\end{array}$ \\
\hline Iztapalapa & 26.140 & 13,1 & $\begin{array}{l}\text { DF-periferia } \\
\text { popular } \\
\text { consolidada }\end{array}$ & 53,5 & 11,8 & 28.695 \\
\hline Ecatepec & 19.180 & 9,6 & $\begin{array}{l}\text { Estado de } \\
\text { México-periferia } \\
\text { popular } \\
\text { consolidada }\end{array}$ & 57,2 & 9,6 & 14.556 \\
\hline Gust. Madero & 13.580 & 6,8 & DF & 48,8 & 16 & 4.196 \\
\hline Coyoacán & 12.880 & 6,5 & DF & 35,9 & 29,6 & 1.975 \\
\hline Nezahualcoyotl & 10.530 & 5,3 & $\begin{array}{l}\text { Estado de } \\
\text { México- periferia } \\
\text { popular } \\
\text { consolidada }\end{array}$ & 44,8 & 12,1 & 400 \\
\hline Alvaro Obregón & 10.140 & 5,1 & DF & 46,5 & 18,8 & 2.278 \\
\hline Ixtapaluca & 9.390 & 4,7 & $\begin{array}{l}\text { Estado de } \\
\text { México: } \\
\text { urbanización } \\
\text { formal (vivienda } \\
\text { social) }\end{array}$ & 45,9 & 11,7 & 12.029 \\
\hline
\end{tabular}

Fuente: Elaboración propia a partir del Censo de población 2000, muestra el 10\% y resultados generales;

Datos Comisión Nacional de Fomento a la Vivienda

* Promedio DF 37,6\%; municipios conurbados $43 \%$

** Promedio 27,3\%; municipios conurbados $20,4 \%$ 


\section{La emigración desde la ciudad central favoreció a la periferia popular consolidada del oriente de la zona metropolitana.}

Casi tres habitantes de cada diez que salieron de las cuatro delegaciones centrales se fueron a instalar en tres importantes unidades político administrativas: Nezahualcóyotl, Ecatepec e Iztapalapa (DF), las cuales comparten un cierto número de puntos comunes y han sido identificadas, por ello mismo, como la "periferia popular consolidada": muy pobladas y completamente urbanizadas, estas tres demarcaciones conocieron en su momento un ritmo de intensa urbanización respectivamente, en los años 1960, 1970 y 1980, tanto por medio de fraccionamientos populares, generalmente de origen irregular (Nezahualcóyotl) como también por la construcción de conjuntos de viviendas de interés social (Ecatepec e Iztapalapa): han recibido una gran cantidad de población de nivel socio-económico muy bajo (Duhau, 2003). Hoy en día, son espacios urbanos muy populares, bien consolidados e identificados de hecho en algunos diagnósticos socio-espaciales de la Ciudad de México como el grupo "medio bajo" (Rubalvaca y Schteingart, 2000).

Estas tres unidades político administrativas de periferia popular consolidada que constituyen un importante destino de emigrantes saliendo de la ciudad central comparten otro punto en común: expulsan a su vez a un gran número de residentes. Iztapalapa, que recibió un número muy elevado de migrantes intrametropolitanos entre los años 1995 y 2000, también expulsó a la mayor cantidad de individuos durante el mismo periodo: 130.000 personas se han ido de esta delegación para otro lugar de residencia en la zona metropolitana. La observación vale para Nezahualcóyotl y Ecatepec (respectivamente, 126.000 y 80.000 emigrantes intra metropolitanos). Esos dos municipios muestran además saldos migratorios negativos con el resto de la zona metropolitana: solamente -14.920 habitantes para la primera, pero -82.199 para la segunda. Cabe mencionar que Nezahualcóyotl incluso entró en una fase de despoblamiento a partir de los años 90 .

Cuando se examinan en detalle cuáles fueron los destinos de los migrantes que salieron de estas delegaciones y municipios de urbanización popular hoy bastante consolidada, se observa que se dieron importantes traslados de población entre esas mismas unidades político administrativas, pero también, un gran número de salidas hacia periferias populares -siempre hacia el oriente de la aglomeración- más lejanas y de urbanización mucho más reciente:

- Por una parte, sectores de urbanización informal e irregular de nivel social económico muy bajo: Chimalhuacán, la Paz, Valle de Chalco... Como ejemplo, Chimalhuacán es uno de los principales destinos para las familias jóvenes que se forman en Nezahualcóyotl (González Alva, 2005; Vega, 2006). Es importante notar que estas unidades político administrativas de urbanización popular ante todo informal que atraen a muchos residentes de la periferia consolidada no constituyen, en cambio, lugares de instalación significativos para los residentes de la ciudad central. No obstante, se produce un traslado de población entre estos tres tipos de espacio: salidas de las cuatro delegaciones centrales alimentan unidades político administrativas de la periferia popular consolidada, las cuales expulsan a su vez un gran número de residentes hacia las periferias más lejanas y más recientes. 
- Por otra parte, periferias populares de urbanización igualmente bastante recientes pero sobre todo formal: municipios que recibieron numerosos programas de viviendas de interés social (cf. supra). A modo de ejemplo, Iztapalapa, Nezahualcóyotl y Ecatepec han expulsado entre 1995 y 2000 a un poco más de 35.000 individuos hacia Ixtapaluca, un municipio de menos de 200.000 habitantes en el año 1995 y en el cual varios asentamientos de gran tamaño han sido construidos entre 1995 y 2000 (Vega, 2003).

Las zonas recientes de producción masiva de vivienda de interés social también atraen a los habitantes del centro.

Los sectores de fuerte producción de viviendas sociales formales (mismos que reciben numerosos habitantes procedentes de la periferia popular consolidada, como lo acabamos de mencionar) constituyen también un destino directo para los individuos que dejan el espacio central: el 12\% (23.290 personas) de estos últimos dejaron de residir en la ciudad central para instalarse en unidades político administrativas que se caracterizan ante todo por este tipo de urbanización.

Si añadimos que Iztapalapa y Ecatepec, grandes destinos de migrantes saliendo de las cuatro delegaciones centrales como ya lo subrayamos, han sido también zonas de muy importante producción de vivienda social formal durante los años 1995-2000, del mismo modo que Gustavo Madero (en el DF), podemos constatar que algunas de las principales zonas de llegada de los individuos que dejaron la ciudad central no son solamente delegaciones y municipios populares consolidados, sino también lugares marcados por una fuerte producción de vivienda de interés social. Se puede indagar entonces la importancia del lazo que existe entre el despoblamiento del centro y la producción de este tipo de vivienda en la Zona Metropolitana de la Ciudad de México.

\section{La ciudad central, un espacio en proceso de recomposición social}

Nuevos residentes en la ciudad central: un perfil que contrasta con el de los emigrantes.

El perfil de individuos que llegaron a las cuatro delegaciones centrales contrasta en muchos puntos con el de los emigrantes que salieron de este espacio, cualquiera que sea su destino. Muchas de estas diferencias no constituyen ninguna sorpresa. Es el caso, por ejemplo, del tipo de tenencia de vivienda. Más de la mitad de los individuos que salieron de la ciudad central entre 1995 y 2000 son dueños de su vivienda en 2000 (y la proporción es mayor para los que se instalaron en el Estado de México). En cambio, una mayoría de personas que migraron del DF hacia el área central no son propietarios en 2000. Este contraste se explica fácilmente, siendo el centro la parte de la ciudad donde la tasa de propiedad es la más baja. En 2000, ésta era de $58,7 \%$, un promedio muy por debajo del resto del DF $(74,3 \%)$ o de la parte de la aglomeración situada en el Estado de México (76,3\%). Las salidas de la ciudad central suelen corresponder en gran parte a migraciones de acceso a la propiedad; las entradas en este espacio lo son en menor medida. 
Consecuentemente, un gran número de individuos que salieron del centro viven en el año 2000 en una casa individual, sobre todo si residen en el Estado de México. Esto aplica mucho menos para los que llegaron al DF (46\% viven en departamentos). Los que se instalaron en la ciudad central entre los años 1995-2000 viven mayoritariamente también en departamentos en 2000 (57,4\%). Encontramos sin embargo cerca de una cuarta parte en casa individual, un tipo de vivienda todavía muy presente en la ciudad central.

Más allá de las diferencias esperadas, otras merecen ser mencionadas en cuanto a los perfiles de los salientes y de los entrantes. El tamaño medio de las familias a las cuales pertenecen los individuos que llegaron a la ciudad central es más reducido que para los que salieron. Se trata aquí de un fenómeno poco sorprendente, siendo alimentadas las dinámicas de reurbanización de los centros principalmente por hogares pequeños y hasta unifamiliares (Ogden, 2000).

Sin embargo, el contraste que merece ser subrayado aun más es sin duda de índole socioeconómico. Añadida a algunas de las evoluciones ya mencionadas anteriormente (como el tamaño de las familias, por ejemplo), esta diferencia permitiría confirmar la existencia de una tendencia a una recomposición paulatina del poblamiento de la ciudad central anterior a la implementación de la política de redensificación (que hoy se acusa de provocar tal cambio, contradictorio con los objetivos planteados por el Gobierno del DF en cuanto a la redensificación del área central). Una transformación de la ciudad central de este tipo ya fue señalada para la segunda mitad del siglo XX sin que sea sin embargo formalmente medida (Coulomb, 1995).

Existe por una parte una diferencia de ingreso bastante significativa entre los individuos que entraron a la ciudad central y los que salieron de ésta. El perfil de estos últimos confirma el carácter popular de buena parte de esta emigración, ya subrayado por los destinos. Estos migrantes son mayoritariamente de bajos ingresos. Tienen además un nivel de estudios bajo y este perfil es aún más marcado cuando se mudan del área central hacia el Estado de México que cuando se dirigen hacia el resto del DF. Sin embargo, tampoco se trata de una población muy pobre. Solo poco menos de la mitad pertenecía en el año 2000 a grupos familiares con un ingreso por debajo de cinco salarios mínimos. Es precisamente el umbral por encima del cual se considera que empieza la "clase media baja" y que el acceso a la vivienda de interés social es considerado posible (Boils, 2003). Con ingresos bajos pero no pobres... el perfil socioeconómico de emigrantes del centro explicaría en parte el tipo de destino que privilegian: las colonias de la periferia popular bien consolidada; las de vivienda social formal, recientes; y en cambio, muy poco las nuevas periferias populares informales.

El nivel de educación de los emigrantes indica un fenómeno comparable: es poco elevado en su conjunto. El mayor número acabó la primaria (6 ańos de estudio), y, cuando más, la segundaria (12 años de estudio); más de un tercio de los emigrantes tiene sin embargo un nivel de estudio que es inferior o igual a seis ańos de estudio. La migración es selectiva igualmente en este plano: en dirección del Estado de México, se caracteriza por una población menos educada.

En cambio, los individuos que llegaron a la ciudad central, procedentes del DF, tenían ingresos promedios que equivalían a más del doble de los que salieron de este espacio hacia el Estado 
de México. El 62\% de los residentes del área central que emigraron hacia el resto de la aglomeración pertenecían en 2000 a familias con sueldo per capita inferior o igual a dos salarios mínimos; los inmigrantes son solamente el $51 \%$ en esta categoría. La proporción de ingresos per capita superiores a cinco salarios mínimos es más elevada para los inmigrantes que para los emigrantes. Dentro de esta categoría, los individuos que pertenecen a familias con 15 salarios mínimos o más (nivel socio-económico alto) representan sin embargo casi la misma proporción, con una leve ventaja para los que llegan: el 10\% de emigrantes contra el 11\% de inmigrantes, una proporción que no es despreciable en ambos casos y que conviene destacar.

Para completar el análisis, cabe mencionar también que los migrantes intrametropolitanos que llegaron a la ciudad central tienen un nivel de educación medio más elevado que los que dejaron este sector. Es entre estos individuos que llegaron a las cuatro delegaciones centrales que encontramos el porcentaje más bajo, con una escolaridad que no excede 6 años (la escuela primaria mexicana) y la más alta proporción con un nivel educativo elevado: entre los que migraron desde el DF, más del 50\% tienen una educación superior a 10 años, el $22,35 \%$ hicieron estudios superiores, contra solamente el $14,6 \%$ para los que salieron del área central.

Instalarse en la ciudad central: la migración más exigente de todas en el plano socio-económico, sea cual sea la procedencia de los individuos.

Más allá de estas diferencias entre los habitantes que dejaron el área central y los que llegaron a vivir en ésta, podemos constatar finalmente, que la instalación en este espacio corresponde a la migración más exigente de todas: este sector de la ciudad capta, para todas las procedencias, a los individuos con nivel socio-económico más elevado.

El caso de la migración a la zona metropolitana desde la provincia es el más ilustrativo. Estos migrantes tienen niveles de ingreso y de educación más bajos que los migrantes intrametropolitanos. Pero dentro de estos, los que llegaron al centro son más educados y con ingresos menos bajos que los que se instalaron en el DF; las personas que se dirigieron hacia un municipio conurbado del Estado de México teniendo un nivel socio-económico y educativo aun más bajo.

Existe por lo tanto una graduación en las migraciones: la que tiene como destino el área central de la ciudad es la más exigente de todas, seguida por la que se dirige hacia el resto del DF y finalmente, por la que llega al Estado de México. La movilidad residencial interna a la ciudad central, que no se mencionó hasta ahora en este trabajo, es la más elitista. Los individuos que cambiaron de delegación para asentarse en el área central son los que tienen el nivel de estudios y de ingresos más elevados de todos los migrantes (ver figuras 4 y 5 ).

Mencionábamos anteriormente una migración intrametropolitana a partir del centro "a dos velocidades": los ingresos y los niveles educativos mas bajos se dirigen hacia el Estado de México, mientras los individuos que se instalan en el DF tienen niveles de ingreso y de educación superiores; es en realidad la existencia de una ciudad "a tres velocidades" que surge del análisis de las movilidades residenciales intraurbanas: un espacio central que atrae a las personas con nivel socio-económico más elevado, seguido por el DF, y luego, para los más pobres y menos 
educados, por los municipios conurbados del Estado de México; un esquema identificado en otros contextos (Donzelot, 2004).

Figura 4

INGRESO PROMEDIO EN 2000 DE LOS MIGRANTES

SEGÚN EL TIPO DE MIGRACIÓN REALIZADO:

DENTRO Y HACIA LA CIUDAD DE MÉXICO (MIGRACIÓN 1995-2000)

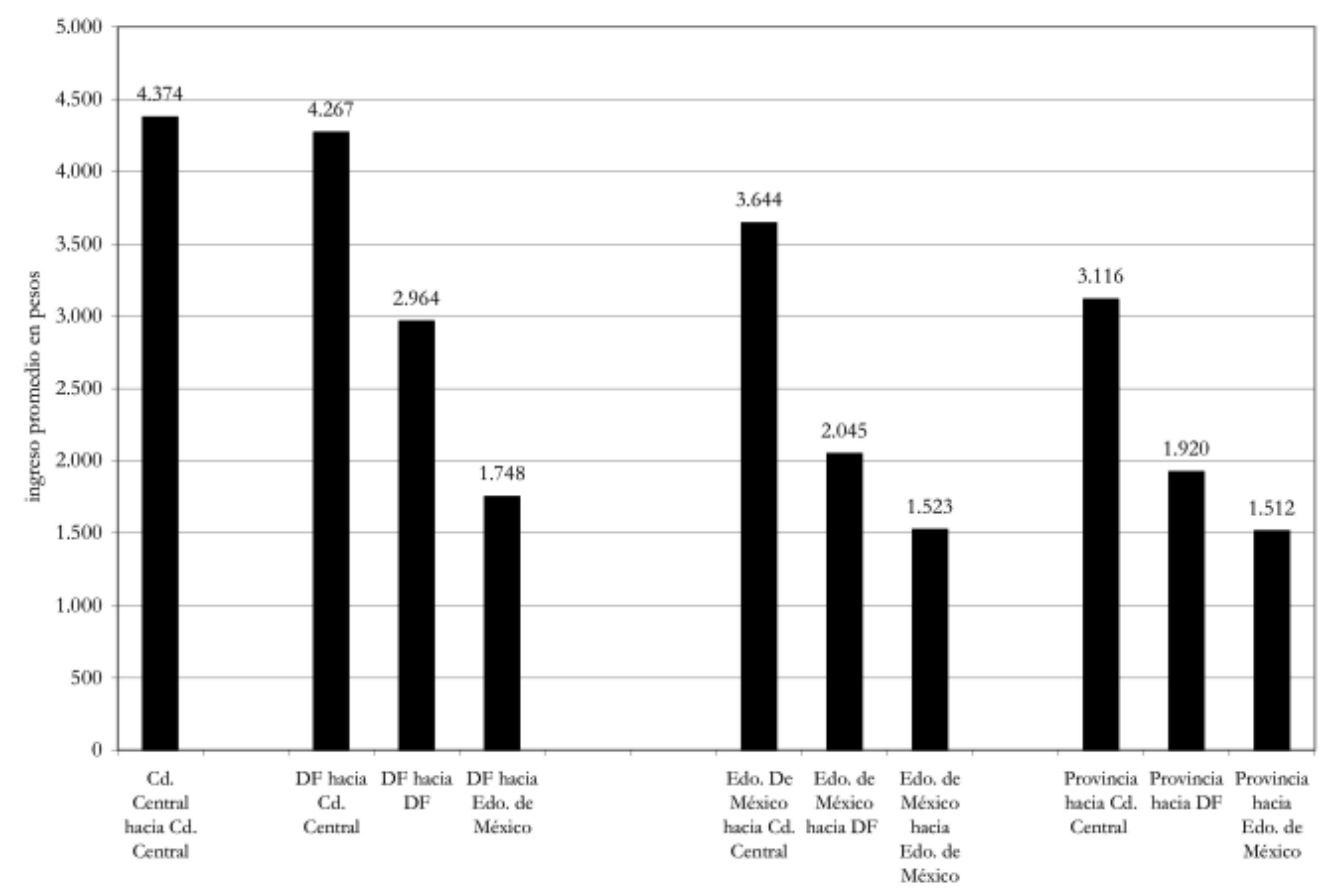

Fuente: Elaboración propia a partir de censo de población 2000, muestra al $10 \%$. 
Figura 5

NIVEL EDUCATIVO PROMEDIO EN 2000 DE LOS MIGRANTES SEGÚN EL TIPO DE MIGRACIÓN REALIZADO: DENTRO Y HACIA LA CIUDAD DE MÉXICO (MIGRACIÓN 1995-2000)

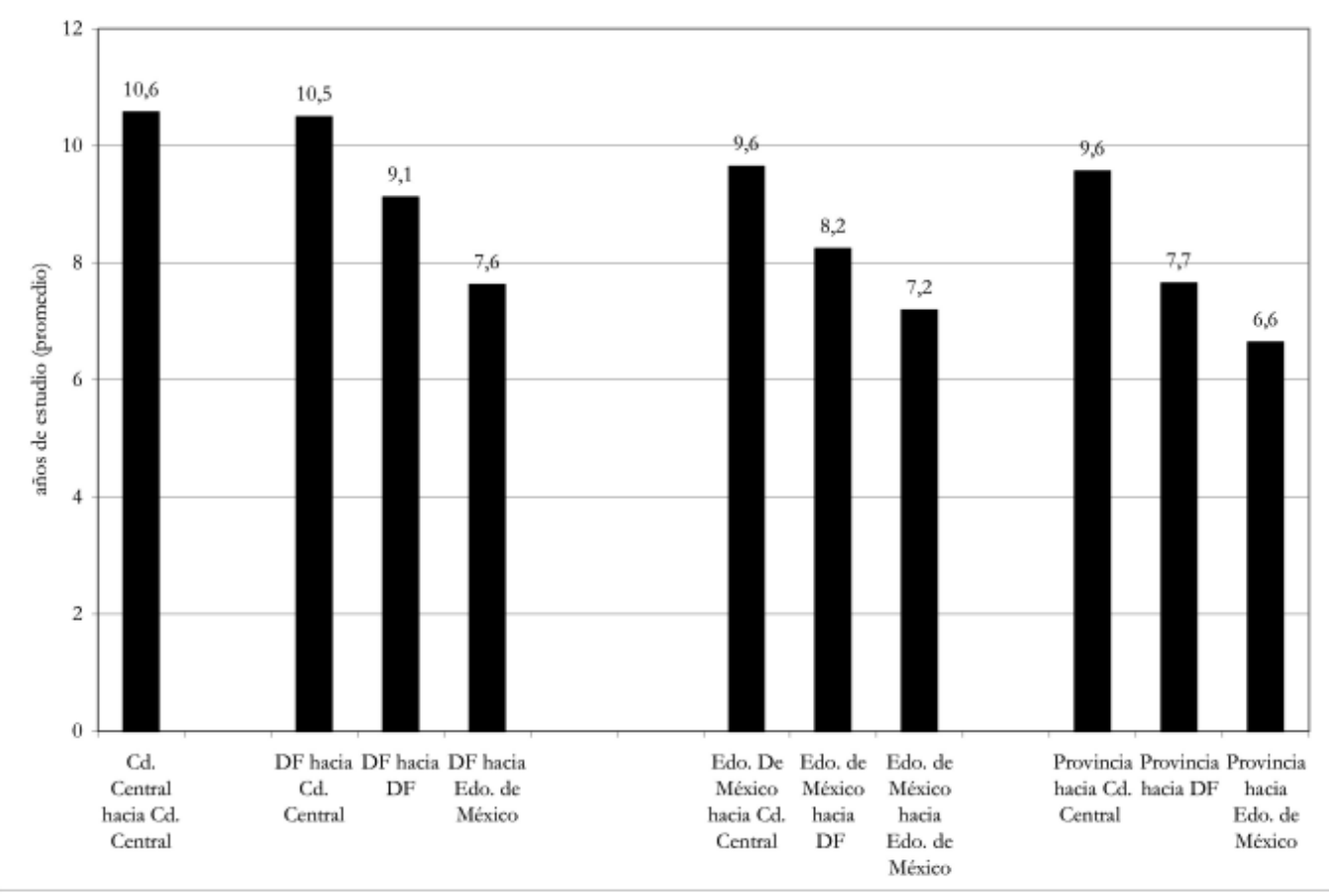

Fuente: Elaboración propia a partir de censo de población 2000, muestra al 10\%.

\section{Conclusión: la pertinencia de la política de redensificación en el contexto migratorio preexistente}

La política urbana planteada para redensificar el sector central de la Ciudad de México fijaba un objetivo muy general: promover el arraigo de la población residente y atraer a nuevos habitantes; al mismo tiempo, "facilitar el acceso a la vivienda para las familias con bajo nivel de recursos" (PGDUDF, 2003). Tal y como lo hemos destacado, estas metas no derivaban de ningún diagnóstico previo con respecto al despoblamiento del centro. La opción de otorgar una connotación social al reciclaje urbano de la ciudad central no provenía de una observación fina de los movimientos migratorios hacia y desde este espacio: reflejaba más bien la voluntad política de un gobierno "de izquierda» recientemente electo (cuyo lema muy explícito no era otro que "los pobres primero") que decidió implementar en el Distrito Federal un plan de acción muy voluntarista en materia de vivienda popular (150.000 acciones durante el sexenio), a través de su Instituto de la Vivienda (Tamayo, 2007).

Aunque no fue el resultado de una caracterización de la emigración a partir de la ciudad central, esta opción social estaba en total congruencia con los procesos en curso: tal y como se explicó, fueron sobre todo los individuos con bajo nivel socio económico los que dejaron de vivir en el área central en el periodo anterior a la política de redensificación, atraídos por las posibilidades de acceder a una vivienda en propiedad en las periferias populares consolidadas o más recientes, de 
urbanización formal, y sin lugar a dudas, también expulsados por un proceso de recomposición social «hacia arriba» del centro (sutil por cierto, pero real). La idea de producir viviendas económicas en este espacio fue por lo tanto muy pertinente para limitar el despoblamiento de este espacio. Los numerosos conjuntos habitacionales populares edificados en la ciudad central gracias a la acción del Instituto de la Vivienda, o incluso, de promotores privados, habrán permitido, sin lugar a dudas, que muchos hogares "cambien de casa pero no de barrio", tal y como lo planteó en su época un estudio del ambicioso programa de renovación habitacional popular implementado en el centro de la Ciudad de México después de los terremotos de 1985 (Connolly, Duhau y Coulomb, 1991).

Sin embargo, podemos preguntarnos si estos esfuerzos fueron suficientes. Por una parte, durante el sexenio de Vicente Fox (2000-2006), y al mismo tiempo que se planteaba une estrategia de repoblamiento para el centro del DF, la producción de viviendas de interés social en grandes conjuntos ubicados en periferias lejanas se intensificó mucho, en el contexto de una planificación urbana en los municipios conurbados del Estado de México que se adaptó a la coyuntura. Esta oferta habitacional (ańadida a la de los fraccionamientos irregulares que siguieron proliferando) ha contribuido a atraer muchos hogares, mismos que la política de redensificación buscaba retener. Por otra parte, dado que no se previó ningún mecanismo de control de los precios del suelo para acompañar el proceso de reciclaje urbano en el centro de la ciudad, las posibilidades de construir viviendas económicas en este espacio se han agotado rápidamente. El concentrar la demanda por terrenos en un sector reducido de la ciudad (cabe recordar que la estrategia de redensificación se basó en el casi congelamiento de las posibilidades de edificar nuevos conjuntos habitacionales en el resto del DF, mientras se facilitaba en la ciudad central) provocó importantes alzas en los precios de venta de los predios (Benliure, 2005). Finalmente, si bien los promotores privados lograron construir viviendas de interés social en el centro, el costo de éstas fue y es todavía superior al de la periferia. La existencia de una oferta masiva de viviendas de interés social de tipo casa unifamiliar en esta parte de la zona metropolitana (cuando, en el centro, se ofrecen solo departamentos) no favoreció, desde lejos, que los hogares modestos se quedaran en el centro. Dada la naturaleza y las características de las migraciones intraurbanas a partir de la ciudad central, solo una estrategia urbana global y coherente a nivel de toda la metrópoli podía pretender limitar el despoblamiento de esta área.

\section{Referencias bibliográficas}

Benliure, P. (2005). Modificaciones al marco normativo del Distrito Federal en la edificación de viviendas, 2000-2005. Ponencia presentada en el Vo Seminario de Suelo Urbano "La redensificación de la ciudad central a debate: ¿para qué, para quién, cómo?, PUEC/UNAM y Lincoln Institute of Land Policy, México DF, México, 29-30 septiembre de 2005.

Boils, G. (2004). El Banco Mundial y la política de vivienda en México. Revista Mexicana de Sociología, 66, 2, 345-367.

Connolly, P.; E. Duhau \& R. Coulomb (1991). Cambiar de casa pero no de barrio. Estudios sobre la reconstrucción en la Ciudad de México. México DF: CENVI/UAM-Azcapotzalco.

Contreras, Y. (2005). Dinámica inmobiliaria en el programa de repoblamiento: un análisis a los efectos urbanos y sociales. Santiago de Chile: Pontificia Universidad Católica de Chile.

Coulomb, R. (1995). Habitat locatif populaire et dynamiques urbaines dans la zone métropolitaine de México. Tesis de doctorado en urbanismo, Instituto de Urbanismo de París, Universidad París XII, Francia. 
Donzelot, J. (2004). La ville à trois vitesses: relégation, périurbanisation, gentrification. Esprit, 303, 14-39.

Duhau, E. (2003). División social del espacio metropolitano y movilidad residencial. Papeles de Población, 36, 161-210.

Garza, G. (2000). La Ciudad de México en el fin del segundo milenio. México DF: El Colegio de México.

González Alva, R. (2005). Intenciones de migración y de movilidad residencial en Chimalhuacán. Tesis de maestría en estudios urbanos, El Colegio de México, México.

Huamán, E. (2005). Políticas de la acción gubernamental en la regularización territorial de la Zona Metropolitana de la Ciudad de México; 1970-2002. Ponencia presentada en el 1er Congreso Nacional de Suelo Urbano, PUEC/UNAM y Lincoln Institute of Land Policy, México DF, México, 23-25 noviembre 2005.

Marcadet, Y. (2007). Habitar en el centro de la ciudad de México. Prácticas espaciales en la Santa María la Ribera. Alteridades, 17, 34, 39-55.

Negrete, M. A. (2000). Migración. En G. Garza (Ed.), La Ciudad de México en el fin del segundo milenio. México DF: El Colegio de México.

Ogden, P. (2000). Households, reurbanization and the rise of living alone en the principal french cities, 1975-1990. Urban Studies, 37, 2, 367-390.

Paquette, C. (2005). La reconquête du centre de Santiago du Chili: un nouveau modèle pour la récupération des centres historiques d'Amérique latine? Cahiers des Amériques latines, 47, 151-165.

Paquette, C. (2006). Des habitants pour le centre historique? México face à l'un des défis majeurs de la réhabilitation. En H. Rivière d'Arc \& M. Memoli, Vivre dans le centre des villes, un pari urbain en Amérique Latine. Paris: Armand Colin.

Paquette, C. (2008). El Metrobús en el contexto de la redensificación urbana: implicaciones y oportunidades. En J. L. Lezama \& C. Salazar, Construir ciudad. Un análisis multidimensional para los corredores de transporte en la Ciudad de México (pp. 195-240). México DF: El Colegio de México.

Programa General de Desarrollo Urbano del Distrito Federal, PGDUDF (2003). Gaceta oficial del Distrito Federal, México.

Puebla Cadena, C. (2002). Del intervencionismo estatal a las estrategias facilitadoras: los cambios en la política de vivienda en México, 1972-1994. México DF: El Colegio de México.

Rubalvaca, R. M. \& Schteingart, M. (2000). Segregación socio espacial. En G. Garza(Ed.), La Ciudad de México en el fin del segundo milenio (pp. 287-296). México DF: El Colegio de México.

Tamayo, S. (2007). Los desafíos del Bando 2. Evaluación multidimensional de las politicas habitacionales en el Distrito Federal 2000-2006. México DF: GDF/SEDUVI/ UACM.

Turner, J. (1968). Settlements patterns and urban development in modernizing cities. Journal of the American Institute of Planners, 34, 354-363.

Vega, A. L. (2003). Urbanización explosiva de la periferia. Ciudades, 57, 57-61.

Vega, A. L. (2006). Mutations du système du logement dans un quartier à développement spontané après la régularisation de la propriété de la terre. Cas de Netzahualcoyotl, banlieue Est de México. Tesis de doctorado en urbanismo, Instituto de Urbanismo de París, Universidad París XII, Francia. 